\title{
The Effects of the Nintendo Wii Exercise Program on Chronic Work-related Low Back Pain in Industrial Workers
}

\author{
Ji-Hyuk Park, OT, PhD ${ }^{1)}$, Sang-Heon Lee, OT, PhD ${ }^{2}{ }^{*}$, Dae-Sik Ko, PT, MPH ${ }^{3)}$ \\ 1) Department of Occupational Therapy, College of Health Science, Yonsei University \\ 2) Department of Occupational Therapy, College of Medical Science, Soon Chun Hyang University: \\ 646 Eupnae-ri, Shinchang-myeon, Asan-si, Chungnam 336-745, South Korea. TEL: +82 41-530-3089, \\ FAX: +82 41-530-3089 \\ 3) Department of Health Science, Graduate School, Chosun University
}

\begin{abstract}
Purpose] The purpose of this study was to investigate the effects of a Nintendo Wii exercise program on chronic work-related LBP compared with stability exercise. [Methods] Twenty-four workers participated in this study. All of the participants were diagnosed with chronic LBP by a physician. Participants were randomly assigned to three groups: a control group (CG), lumbar stabilization exercise group (LSE), and Nintendo Wii exercise group (NWE). Participants were treated 3 times a week for 8 weeks. Each session lasted 30 minutes. [Results] The results demonstrated that exercise programs improved significantly physical functions related to LBP. In health-related QOL, the Nintendo Wii exercise program significantly improved both the mental and physical health composites, but other groups had significant improvement only in the physical health composite. [Conclusion] The Nintendo Wii exercise program could be a biopsychosocial intervention for work-related LBP in factory workers.

Key words: Low back pain, Exercise, Nintendo Wii
\end{abstract}

(This article was submitted Mar. 4, 2013, and was accepted Mar. 29, 2013)

\section{INTRODUCTION}

Work-related low back pain (LBP) is a common workrelated musculoskeletal disorder. In Korea, work-related low back pain makes up $36.7 \%$ of the total reimbursement from industrial accident compensation insurance ${ }^{1)}$. In Western countries, LBP is one of the biggest single reasons for absence from work ${ }^{2)}$. The annual prevalence of LBP has been reported at $38 \%$ across the globe ${ }^{3)}$. LBP commonly attenuates within weeks, but $24-50 \%$ of cases may recur within 1 year ${ }^{4}$. Chronic LBP decreases productivity in the workplace because the recurrence rate is over $60 \%{ }^{5}$. Thus, treatment of chronic LBP is important in work populations.

Chronic LBP induces various problems, including physical, psychosocial, and quality of life features ${ }^{5,6}$. Chronic LBP reduces muscle strength, endurance, flexibility, and balance ability. Among psychological aspects, depression is one of the most common symptoms in chronic LBP ${ }^{7)}$. Psychological symptoms include depression, anxiety, and low self-efficacy, which negatively affect quality of life (QOL) ${ }^{8)}$. Exercise is the main component in treating chronic LBP, but repetitive exercise for strengthening has limitations in increasing motivation for exercise programs and for treating psychosocial symptoms.

Recently, the Nintendo Wii exercise program has been used for therapeutic effect in various populations ${ }^{9,10)}$. The

${ }^{*}$ To whom correspondence should be addressed. E-mail: sangheon@sch.ac.kr
Nintendo Wii console is an instrument of biofeedbackbased exercise. A user controls a game character in a virtual environment using a remote controller with a motion sensor. A user performs similar movements to real sports activities such as swinging a tennis racket or rowing a boat. The Nintendo Wii is commercially available and inexpensive. Game components motivate participants be involved in an exercise program through a sense of fun. A previous study reported that the Nintendo Wii exercise program improved upper extremity function in stroke patients ${ }^{10)}$. The Nintendo Wii exercise program has been shown to improve cognitive function, balance ability, and visual perception in various populations ${ }^{11,12)}$. However, there have been few studies on the effect of the Nintendo Wii exercise program in chronic LBP.

The purpose of this study was to investigate the effect of the Nintendo Wii exercise program on chronic work-related LBP compared to stability exercise. The hypothesis of this study was that the Nintendo Wii exercise program would significantly improve health-related quality of life (QOL) as well as physical function, including core strength, flexibility, endurance, and balance. Health-related QOL includes mental health and emotional factors.

\section{SUBJECTS AND METHODS}

Twenty-four workers from the $\mathrm{K}$ tire factory participated in this study. The inclusion criteria were as follows: 1) diagnosed with chronic LBP by a physician; 2) over 3 
Table 1. General characteristics of subjects

\begin{tabular}{lccc}
\hline & CG $(\mathrm{n}=8)$ & LSE $(\mathrm{n}=8)$ & NWE $(\mathrm{n}=8)$ \\
\hline Age & $45.50 \pm 5.34$ & $43.37 \pm 5.42$ & $44.12 \pm 5.48$ \\
Height & $169.43 \pm 2.07$ & $170.57 \pm 3.23$ & $170.48 \pm 2.87$ \\
Weight & $68.90 \pm 5.78$ & $72.50 \pm 7.09$ & $71.92 \pm 7.1$ \\
Years of service & $18.75 \pm 7.38$ & $16.00 \pm 6.88$ & $17.00 \pm 7.59$ \\
\hline
\end{tabular}

Mean \pm SD. * $\mathrm{p}<0.05$. CG, control group; LSE, lumber stabilization exercise; NWE, Nintendo Wii exercise

months with LBP; and 3) no experience with surgical treatments for disc herniation, spina bifida, or spinal stenosis. The average age was 44 years old. The average length of service was 17 years (Table 1). Participants were randomly assigned to three groups: a control group (CG), lumbar stabilization exercise group (LSE), and Nintendo Wii exercise group (NWE). There were no significant differences among groups in height, weight, age, or years of service ( $p>0.05$ ).

In each group, physical therapy was provided using physical agent modalities such as a hot pack (30 min), interferential current therapy (15 min), and deep heat with ultrasound (5 min). Subsequently, an additional exercise program was provided in the LSE and NWE groups, but not in the control group. Participants were treated 3 times a week for 8 weeks. Each session lasted 30 minutes. Stabilization exercise included 7 positions based on the back bridge, hands and knees, and side bridge as follows ${ }^{13)}$ : 1) standard back bridge, 2) back bridge with alternate leg straight, 3) back bridge with one leg, 4) standard hands and knees, 5) hands and knees with alternate arm straight forward, 6) hands and knees with alternate leg straight backward, and 7) side bridge. Participants in the LSE group were asked to maintain each position for 15 seconds for 3 sets. The Nintendo Wii sports program was used for the Nintendo Wii exercise program, including the wakeboard, Frisbee dog, jet ski, and canoe games. Participants in the NWE group controlled a virtual character on the screen by swinging, rowing, and tilting remote controllers with motion sensors. Participants chose which Nintendo Wii sports program they performed and took a 2 minute break every 10 minutes.

Before and after 8 weeks of intervention, the participants of each group were evaluated for pain, back strength, balance ability, and health-related QOL with the following tools; visual analog scale (VAS) for pain ${ }^{14)}$, isometric lifting strength for back strength ${ }^{15}$, 16), One-legged Stand Test for balance ability ${ }^{16,17)}$, and the RAND-36 Health Status Inventory (RAND-36) for health-related $\mathrm{QOL}^{18)}$. Back strength was defined as the maximum isometric lifting weight ${ }^{15}$. Balance was evaluated by measuring how long the subject could stand on one leg ${ }^{17)}$. The RAND-36 includes a physical and mental health summary score derived from the 36-item Short-Form Health Survey (SF-36) items. The physical health category includes physical functioning, role limitations resulting from physical health, pain, and general health perceptions. The mental health category includes emotional well-being, role limitations resulting from emotional problems, social functioning, and energy/fatigue. One-way ANOVA was used to test group differences in pre-
Table 2. Changes in pain and physical function

\begin{tabular}{lccc}
\hline Category & Group & Pre-test & Post-test \\
\hline \multirow{4}{*}{ Pain } & CG $(\mathrm{n}=8)$ & $6.50 \pm 1.30$ & $5.75 \pm 0.88$ \\
& LSE $(\mathrm{n}=8)$ & $6.62 \pm 0.74$ & $4.87 \pm 0.83^{*}$ \\
& $\mathrm{NWE}(\mathrm{n}=8)$ & $6.75 \pm 1.38$ & $5.87 \pm 1.12^{*}$ \\
\hline \multirow{2}{*}{ Back } & $\mathrm{CG}(\mathrm{n}=8)$ & $53.73 \pm 22.07$ & $55.58 \pm 23.20^{*}$ \\
strength & $\mathrm{LSE}(\mathrm{n}=8)$ & $54.43 \pm 12.72$ & $60.16 \pm 13.58^{*}$ \\
& $\mathrm{NWE}(\mathrm{n}=8)$ & $54.48 \pm 10.31$ & $57.81 \pm 9.07^{*}$ \\
\hline \multirow{2}{*}{ Balance } & $\mathrm{CG}(\mathrm{n}=8)$ & $8.62 \pm 3.20$ & $10.75 \pm 3.61^{*}$ \\
ability & $\mathrm{LSE}(\mathrm{n}=8)$ & $8.50 \pm 3.07$ & $11.37 \pm 3.62^{*}$ \\
& $\mathrm{NWE}(\mathrm{n}=8)$ & $8.75 \pm 1.83$ & $10.62 \pm 3.73$ \\
\hline
\end{tabular}

Mean \pm SD. * $p<0.05$. CG, control group; LSE, lumber stabilization exercise; NWE, Nintendo Wii exercise

test values. Paired t-test was used to compare pre- and posttest values within each group.

\section{RESULTS}

Pain decreased significantly in the LSE and NWE groups, but not in the control group $(\mathrm{p}<0.05)$ (Table 2$)$. All groups demonstrated a significant increase in back strength $(p<0.05)$ (Table 2). The control and LSE groups showed a significant improvement in balance, but the NWE group did not. In the physical health composite of QOL, all three groups improved significantly in pain and general health $(p<0.05)$ (Table 3$)$. Only the LSE group improved significantly in physical functioning and role limitations due to physical health $(\mathrm{p}<0.05)$ (Table 3$)$. In the mental health composite of QOL, the control and LSE groups showed no significant changes, but the NWE group improved significantly in terms of role limitations due to emotional problems, energy/fatigue, and emotional well-being $(p<0.05)$ (Table 4). There were no significant differences in the pretest values among groups $(\mathrm{p}>0.05)$.

\section{DISCUSSION}

Work-related musculoskeletal disorders are a significant issue in industry. LBP is one of the most common workrelated disorders in the world ${ }^{3)}$. The most common therapy for LBP includes physical agent modalities and stabilization exercise $^{13)}$. However, these common approaches for LBP do not consider mental and cognitive aspects that are important factors in LBP in addition to physical factors. Recently, the Nintendo Wii exercise program has been employed in various populations, and positive effects have been reported with regard to physical and mental health ${ }^{11,19)}$. However, there have been few studies on the effects of the Nintendo Wii exercise program on work-related LBP. Thus, the purpose of this study was to investigate the effects of the Nintendo Wii exercise program on work-related low back pain in factory workers. Participants were randomly assigned to three groups: control, stabilization exercise program, and Nintendo Wii exercise program. The results demonstrated that exercise programs significantly improved physical function-related LBP. In health-related QOL, the Nintendo 
Table 3. Changes of QOL in the physical health composite of the RAND-36

\begin{tabular}{lrll}
\hline Category & Group & Pre-test & Post-test \\
\hline \multirow{4}{*}{ Physical functioning } & CG $(\mathrm{n}=8)$ & $57.50 \pm 26.32$ & $58.12 \pm 26.98$ \\
& LSE $(\mathrm{n}=8)$ & $48.75 \pm 19.59$ & $57.50 \pm 17.92^{*}$ \\
& $\mathrm{NWE}(\mathrm{n}=8)$ & $53.12 \pm 13.34$ & $54.37 \pm 12.37$ \\
\hline \multirow{3}{*}{ Role limitations due to physical health } & $\mathrm{CG}(\mathrm{n}=8)$ & $67.25 \pm 20.87$ & $68.12 \pm 21.66$ \\
& $\mathrm{LSE}(\mathrm{n}=8)$ & $71.12 \pm 9.89$ & $81.50 \pm 13.56^{*}$ \\
& $\mathrm{NWE}(\mathrm{n}=8)$ & $69.62 \pm 14.75$ & $73.50 \pm 16.65$ \\
\hline \multirow{3}{*}{ Pain } & $\mathrm{CG}(\mathrm{n}=8)$ & $30.00 \pm 14.14$ & $36.25 \pm 10.60^{*}$ \\
& $\mathrm{LSE}(\mathrm{n}=8)$ & $42.50 \pm 19.82$ & $57.50 \pm 12.81^{*}$ \\
& $\mathrm{NWE}(\mathrm{n}=8)$ & $32.50 \pm 19.08$ & $40.00 \pm 14.14^{*}$ \\
\hline \multirow{3}{*}{ General health } & $\mathrm{CG}(\mathrm{n}=8)$ & $45.00 \pm 18.12$ & $50.00 \pm 17.32^{*}$ \\
& $\mathrm{LSE}(\mathrm{n}=8)$ & $50.00 \pm 12.24$ & $64.37 \pm 11.78^{*}$ \\
& $\mathrm{NWE}(\mathrm{n}=8)$ & $41.25 \pm 18.85$ & $44.37 \pm 18.60^{*}$ \\
\hline
\end{tabular}

Mean \pm SD. ${ }^{*} \mathrm{p}<0.05$. CG, control group; LSE, lumber stabilization exercise; NWE, Nintendo Wii exercise

Table 4. Changes of QOL in the mental health composite of the RAND-36

\begin{tabular}{lrcc}
\hline Category & Group & Pre-test & Post-test \\
\hline \multirow{4}{*}{ Role limitations due to emotional problem } & CG $(\mathrm{n}=8)$ & $71.00 \pm 19.84$ & $73.00 \pm 15.36$ \\
& LSE $(\mathrm{n}=8)$ & $78.00 \pm 13.23$ & $80.25 \pm 10.83$ \\
& $\mathrm{NWE}(\mathrm{n}=8)$ & $68.75 \pm 15.90$ & $81.12 \pm 10.84^{*}$ \\
\hline \multirow{3}{*}{ Energy/Fatigue } & $\mathrm{CG}(\mathrm{n}=8)$ & $62.75 \pm 22.77$ & $62.00 \pm 16.85$ \\
& $\mathrm{LSE}(\mathrm{n}=8)$ & $68.87 \pm 15.51$ & $70.50 \pm 10.92$ \\
& $\mathrm{NWE}(\mathrm{n}=8)$ & $58.00 \pm 23.68$ & $68.12 \pm 17.26^{*}$ \\
\hline \multirow{3}{*}{ Emotional wellbeing } & $\mathrm{CG}(\mathrm{n}=8)$ & $70.62 \pm 7.28$ & $72.50 \pm 5.34$ \\
& $\mathrm{LSE}(\mathrm{n}=8)$ & $65.00 \pm 9.25$ & $66.87 \pm 7.98$ \\
& $\mathrm{NWE}(\mathrm{n}=8)$ & $63.12 \pm 6.51$ & $73.12 \pm 7.52^{*}$ \\
\hline \multirow{3}{*}{ Social functioning } & $\mathrm{CG}(\mathrm{n}=8)$ & $59.50 \pm 19.69$ & $59.62 \pm 18.74$ \\
& $\mathrm{LSE}(\mathrm{n}=8)$ & $69.00 \pm 14.82$ & $72.00 \pm 3.93$ \\
& $\mathrm{NWE}(\mathrm{n}=8)$ & $61.00 \pm 18.22$ & $69.12 \pm 9.35$ \\
\hline
\end{tabular}

Mean \pm SD. $* \mathrm{p}<0.05$. CG, control group; LSE, lumber stabilization exercise; NWE, Nintendo Wii exercise

Wii exercise program showed significant improvements in both the mental and physical health composites, but other groups showed significant improvement only in the physical health composite.

Exercise programs are essential to reducing pain and improving QOL in work-related LBP. In the control group, no exercise program was provided outside of pain control approaches such as hot pack, ICT, and ultrasound. These physical agent modalities reduce pain physiologically, but there was no significant change demonstrated in this study because daily work causes cumulated LBP. The short-term effect of physical agent modalities did not significantly reduce work-related LBP. Although both stabilization exercise and Nintendo Wii exercise significantly reduced work-related LBP, stabilization exercise increased muscle strength more than Nintendo Wii exercise because stabilization exercise focused more on the low back muscles compared with the Nintendo Wii exercise. Additionally, only stabilization exercise significantly increased balance. The stabilization exercise program demonstrated more effective increases in physical function than the Wii exercise program. Previous studies have reported that stabilization exercises for the low back significantly improved the strength of the low back muscles, thus reducing $\mathrm{LBP}^{13)}$. However, a physical exercise program only did not improve return to work $^{20)}$. In addition to physical function, LBP decreases the mental and physical aspects of health-related QOL.

The Nintendo Wii exercise program significantly improved both the physical and mental composites of healthrelated QOL. In previous studies, LBP has been shown to affect not only physical function, but also aspects of mental health ${ }^{5,8)}$. For example, chronic LBP is related to depres$\operatorname{sion}^{21)}$. In this study, stabilization exercise did not significantly improve the mental health composite of health-related QOL. The Nintendo Wii exercise program influenced mental health as well as physical health. Other studies have demonstrated that rehabilitation programs using a Nintendo Wii console improved mental and cognitive functions in various populations, which may be related to the mental health aspects of health-related $\mathrm{QOL}^{11,19)}$. The gaming 
components of Nintendo Wii sports encourage participants to enjoy exercise and require mental and cognitive functions, which may be possible reasons for improvement in the mental health composite. However, the Nintendo Wii exercise program was less effective than stabilization exercise in improving the physical function and physical health composites of QOL.

In terms of clinical implications, the Nintendo Wii exercise program could be a biopsychosocial intervention for work-related LBP in factory workers. Recent research supports biopsychosocial approaches for LBP rather than stabilization exercise based on a biomedical model ${ }^{22)}$. Low-back stabilization exercise has been the most commonly prescribed exercise for LBP, but only $8-15 \%$ of LBP was related to a patho-anatomical diagnosis ${ }^{23}$. Although low-back stabilization exercise was previously included in therapeutic interventions for work-related $\mathrm{LBP}^{24}$ ), stabilization exercise did not improve the mental health composite of QOL. The stabilization exercise was based on the biomechanical aspect, not the biopsychosocial aspect. The Nintendo Wii exercise program significantly improved the physical and mental health aspects of QOL. Even though the Nintendo Wii exercise program demonstrated a lower effect on physical function and physical health QOL than stabilization exercise, the Nintendo Wii exercise program improved the mental health composite of QOL, which stabilization exercise barely improved. Future studies are needed on various effects of Nintendo Wii exercise programs, including social and psychological factors, for work-related LBP in factory workers.

\section{ACKNOWLEDGEMENT}

This work was supported by the Soonchunhyang University Research Fund (No. 20110695).

\section{REFERENCES}

1) Korea Occupational Safety and Health Agency: Annual Report. Incheon KOSHA, 2010

2) Andersson GB: Epidemiological features of chronic low-back pain. Lancet, 1999, 354: 581-585. [Medline] [CrossRef]

3) Brage $\mathrm{S}$, Ihlebaek C, Natvig B, et al.: Musculoskeletal disorders as cause of sick leave and disability benefits. Tidsskr Nor Laegeforen, 2010, 130: 2369-2370. [Medline] [CrossRef]

4) Hoy D, Brooks P, Blyth F, et al.: The Epidemiology of low back pain. Best Pract Res Clin Rheumatol, 2010, 24: 769-781. [Medline] [CrossRef]

5) Sterud T, Tynes T: Work-related psychosocial and mechanical risk factor for low back pain: a 3-year follow-up study of the general working popula- tion in Norway. Occup Environ Med, 2013, 70: 296-302. [Medline]

6) Pincus T, Santos R, Breen A, et al.: A review and proposal for a core set of factors for prospective cohorts in low back pain: a consensus statement. Arthritis Rheum, 2008, 59: 14-24. [Medline] [CrossRef]

7) Licciardone JC, Gatchel RJ, Kearns CM, et al.: Depression, somatization, and somatic dysfunction in patients with nonspecific chronic low back pain: results from the Osteopathic Trial. J Am Osteopath Assoc, 2012, 112: 783-791. [Medline]

8) Mok LC, Lee IF: Anxiety, depression and pain intensity in patients with low back pain who are admitted to acute care hospitals. J Clin Nurs, 2008, 17: 1471-1480. [Medline] [CrossRef]

9) Wuang YP, Chiang CS, Su CY, et al.: Effectiveness of virtual reality using Wii gaming technology in children with Down syndrome. Res Dev Disabil, 2011, 32: 312-321. [Medline] [CrossRef]

10) Saposnik G, Teasell R, Mamdani M, et al.: Effectiveness of virtual reality using Wii gaming technology in stroke rehabilitation: a pilot randomized clinical trial and proof of principle. Stroke, 2010, 41: 1477-1484. [Medline] [CrossRef]

11) Pompeu JE, Mendes FA, Silva KG, et al.: Effect of Nintendo Wii-based motor and cognitive training on activities of daily living in patients with Parkinson's disease: a randomised clinical trial. Physiotherapy, 2012, 98: 196-204. [Medline] [CrossRef]

12) Clark R, Kraemer T: Clinical use of Nintendo Wii bowling simulation to decrease fall risk in an elderly resident of a nursing home: a case report. J Geriatr Phys Ther, 2009, 32: 174-180. [Medline] [CrossRef]

13) Imai $\mathrm{A}$, Kaneoka $\mathrm{K}$, Okubo $\mathrm{Y}$, et al.: Trunk muscle activity during lumbar stabilization exercises on both a stable and unstable surface. J Orthop Sports Phys Ther, 2010, 40: 369-375. [Medline]

14) Hawksley H: Pain assessment using a visual analogue scale. Prof Nurse 2000, 15: 593-597. [Medline]

15) Hattori $Y$, Ono $Y$, Shimaoka $M$, et al.: Test-retest reliability of isometric and isoinertial testing in symmetric and asymmetric lifting. Ergonomics, 1998, 41: 1050-1059. [Medline] [CrossRef]

16) Ko DS, Lee CG, Kim GY, et al.: The effect of lumbar stabilization exercise on motor capacity and pain in chronic low back pain workers. J Sports Lesiure Stud, 2009, 35: 1021-1028.

17) Franchignoni F, Tesio L, Martino MT, et al.: Reliability of four simple, quantitative tests of balance and mobility in healthy elderly females. Aging (Milano), 1998, 10: 26-31. [Medline]

18) Hays RD, Sherbourne CD, Mazel RM: The RAND 36-Item Health Survey 1.0. Health Econ, 1993, 2: 217-227. [Medline] [CrossRef]

19) Rosenberg D, Depp CA, Vahia IV, et al.: Exergames for subsyndromal depression in older adults: a pilot study of a novel intervention. Am J Geriatr Psychiatry, 2010, 18: 221-226. [Medline] [CrossRef]

20) Hagen EM, Odelien $\mathrm{KH}$, Lie SA, et al.: Adding a physical exercise programme to brief intervention for low back pain patients did not increase return to work. Scand J Public Health, 2010, 38: 731-738. [Medline] [CrossRef]

21) Ellegaard H, Pedersen BD: Stress is dominant in patients with depression and chronic low back pain. A qualitative study of psychotherapeutic interventions for patients with non-specific low back pain of 3-12 months'duration. BMC Musculoskelet Disord, 2012, 13: 166. [Medline] [CrossRef]

22) O'Sullivan P: It's time for change with the management of non-specific chronic low back pain. Br J Sports Med, 2012, 46: 224-227. [Medline] [CrossRef]

23) Waddell G: The back pain revolution. New York: Churchill Livingstone, 2004

24) Davarian S, Maroufi N, Ebrahimi I, et al.: Trunk muscles strength and endurance in chronic low back pain patients with and without clinical instability. J Back Musculoskelet Rehabil, 2012, 25: 123-129. [Medline] 